\title{
Design of Low-Cost Unmanned Underwater Vehicle for Shallow Waters
}

\author{
W.H. Wang*, X.Q. Chen, A. Marburg, J.G. Chase, C.E. Hann
}

\begin{abstract}
Unmanned underwater vehicles (UUVs) have received worldwide attention and been widely used in various applications. In this paper, a recently developed low cost UUV prototype at the University of Canterbury is introduced, which is designed specifically for shallow water tasks, especially for inspecting and cleaning sea chests of ships for biosecurity purpose. The main hull of the UUV is made of PVC, with a 400mm diameter and 800mm length. External frames mount two horizontal propellers, four vertical thrusters, and power is derived from onboard batteries. The maximum thrust force of up to $10 \mathrm{~kg}$ that is provided by the propellers can generate a forward/backward speed of up to $1.4 \mathrm{~m} / \mathrm{s}$ for the $112 \mathrm{~kg} \mathrm{UUV}$. The vertical thrusters provide depth control with a max thrust force of $20 \mathrm{~kg}$. The UUV is equipped with a range of sensors capable of sensing its instantaneous temperature, depth, attitude and surrounding environment. Costing less than US $\$ 10,000$ for a prototype, it provides an excellent platform for further underwater vehicle development targeting shallow water tasks with a working depth up to $20 \mathrm{~m}$.
\end{abstract}

Index Terms

Unmanned underwater vehicle, low cost, hull design, shallow water inspection

\section{INTRODUCTION}

Unmanned underwater vehicles (UUVs) offer many advantages for performing difficult tasks submerged in water. Although many names are given to these vehicles, such as remotely operated vehicles (ROVs), submersible devices, or remote controlled submarines, to name just a few, the fundamental task for these devices is fairly well defined. They operate in conditions and perform task that humans are not able to do efficiently, or at all [1], [2], [3].

First developed in the 1960's, development was driven by the demand from the US Navy [4], which required them to perform deep sea rescue and salvage operations. Since then, other sectors have realized the potential of such devices for all manner of tasks. The first of these was the oil and gas industry. These companies employed UUVs to reinforce in the development of off shore oil fields [5]. In the 1980's, UUVs came into a new era as they were able to operate at depths well below commercial diver limits. Falling oil prices and a global recession resulted in a stagnant period in terms of UUV development in the mid 1980s. Since then however, UUVs have been developing at a fast rate [6], [7].

Wang, Chen, Chase, and Hann are with the Dept. of Mechanical Engineering, University of Canterbury, Private Bag 4800, Christchurch 8140 , New Zealand (* Correspondence Author. E-mail: wenhui.wang@canterbury.ac.nz).

Marburg is with Geospatial Research Centre (NZ) Ltd, Private Bag 4800, Christchurch 8140, New Zealand. 


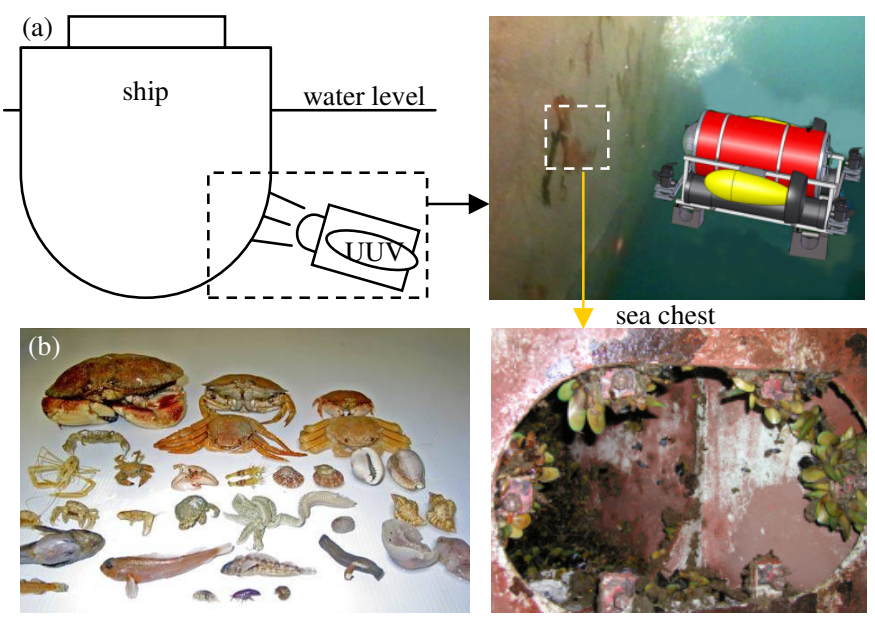

Fig. 1. Unmanned underwater vehicle inspecting and cleaning sea chest of ships. (a) The diagram of the UUV working on the sea chest of the ship. (b) A range of foreign invaders hiding in the sea chest.

UUVs are now being used in a wide range of applications, such as locating historic ship wrecks like the Titanic [8], mapping the sea floor [9]. More mundane applications consist of object detection [10], securing harbours, searching for seamines [11], and, most recently, in scientific applications [12], [13], [14].

As more than half of our oceans are deeper then $3 \mathrm{~km}$, one direction of the UUV developments is to explore deep waters. However, development of such UUVs imposes extreme design specifications for the hardware [15], incurring an unaffordable cost for most labs. By contrast, UUVs for shallow waters recently have gained more attention because of their potentially wide use combined with affordable cost. At the University of Canterbury, a UUV prototype has been recently designed with the primary purpose for inspecting and cleaning the sea chests of ships (Fig. 1a), an application with significant impact in the area of bio-security.

Sea chests are the intake areas in the hulls of ships for seawater used for ballast, engine-cooling and fire-fighting. Grates on the outside of the chests prevent large organisms from being entrained in the water but many smaller organisms (Fig. 1b) survive in the sea chests and are transported around the world creating a bio-security risk. The New Zealand government has placed a high priority on the the development of systems and tools to protect native flora and fauna against invasion by unwanted foreign organisms.

To optimize the knowledge of, and reaction to, this threat, the first task is to inspect the sea chests and collect information about the invaders. Currently, divers are sent to do the job, which has inherent problems, including: i) high cost, ii) unavailability of suitably trained personnel for the number of ships needing inspection, iii) safety concerns, iv) low throughput, and v) unsustainable working time underwater to do a thorough job. To reduce the working load of divers and significantly accelerate inspection and/or treatment, it would be highly desirable and efficient to deploy affordable UUVs to inspect and clean these ship sea chests. Thus, this paper presents a low cost UUV prototype emphasizing the unique design issues and solutions developed for this task, as well as those attributes that are generalizable to similar systems. Control and navigation are being implemented and are thus not 
covered here.

\section{Hull Design}

Fig. 2 shows the UUV prototype (weighing $112 \mathrm{~kg}$, positively buoyant), which consists of basic components, including main hull, two horizontal propellers, four vertical thrusters, two batteries, an external frame, and electronics inside the main hull. This section focuses on the hull design.

There are a number of different ways in which hull design can be approached [16]. These different design methods are typically specific to the situation/task. The main hull must be able to meet a number of key challenges.

Aspects that must be considered during hull design include:

- Pressure and/or depth required

- Operating temperature ranges

- Structural integrity for additions and tapings

- Impact conditions

- Water permeability

- Visual appeal and aesthetics

- Accessibility

- Versatility

- Practicality

- Restrictions for future additions

- Size requirements

- Corrosion and chemical resistance

The foremost design decision is the shape of the hull. Inspired by torpedoes and submarines, a cylindrical hull has been selected. A cylinder has favourable geometry for both pressure (no obvious stress concentrations) and dynamic reasons (minimum drag). To make the hull, three easily accessible materials were compared. The first option is to use a section of highly available PVC storm water pipe. The second option involves having a hull made from a composite material, such as carbon fibre or fibre glass. Mandrel spinning of such a hull will allow more freedom in radial dimensions. The process can in fact incorporate a varying radius along the length resulting in a slender, traditional hull. However, this process requires a large amount of design and set up time. A less desirable third option is to use a section of metal pipe, which is prone to corrosion and has a high weight and cost. As a result, the PVC storm water pipe option was selected.

Two caps were designed to complete the hull, and are attached to each end of the pipe such that they reliably seal the hull. The caps also allow access to the interior for easy repair and maintenance in the field.

The end cap design incorporates an aluminium ring that is permanently fixed to the hull and a removable aluminium plug. The plug fits snugly into the aluminium ring. Sealing is achieved with commercially available O-rings. Sealing directly to the PVC hull would have been more desirable, however this option was not taken for two main reasons. First, PVC does not provide a sealing surface as smooth and even as aluminium and is extremely 


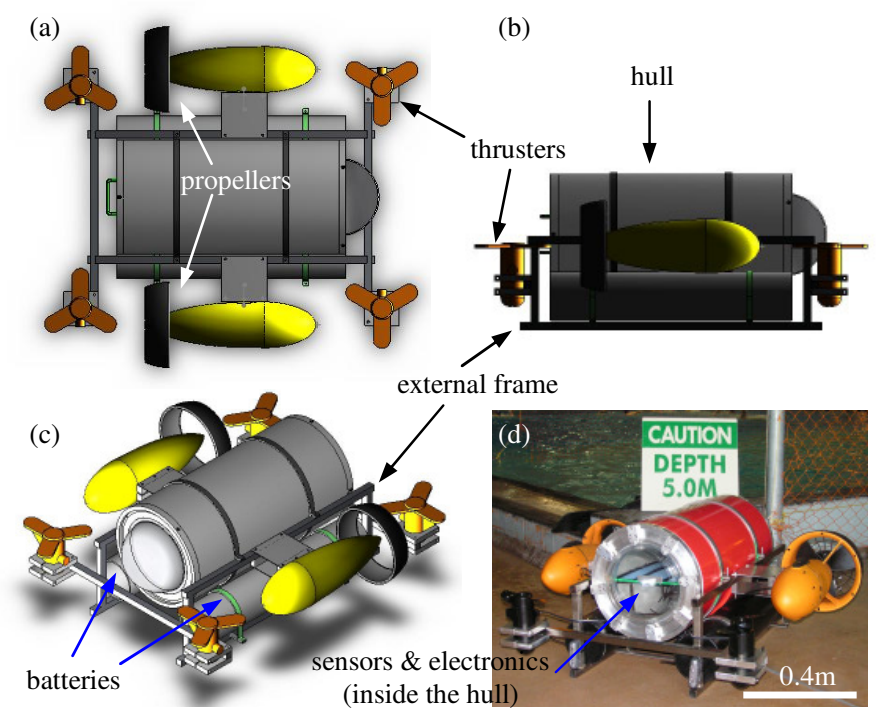

Fig. 2. The hull structure of the vehicle. (a)-(c) Design drawings of the vehicle: (a) Top view. (b) Side view. (c) Isometric view. (d) Real picture of the in-house made vehicle.

hard to machine in this case due to the size of the pipe. Second, the PVC pipe is not perfectly round and subject to significant variability, which would have made any machined aluminum cap subject to poor fit and potential leakage, decreasing reliability.

The design choices made can thus better manage these issues. More specifically, the design is based on selfsealing where greater outside pressures enforce greater connection between the cap, seals, and PVC hull portion. The O-ring seal employed is made of nitrile, which is resistant to both fresh and salt water.

\section{PROPULSION AND STEERING}

The design incorporates 2 horizontal thrusters mounted on both sides of the UUV to provide both forward and backward movement. Yaw is provided by operating the thrusters in opposing directions. The thrusters are $12 \mathrm{~V}$ dive scooters (Pu Tuo Hai Qiang Ltd, China) that have a working depth of up to $20 \mathrm{~m}$.

The dive scooters are lightly modified to enable simple attachment to the external frame of the UUV. The thruster mounts consist of two aluminium blocks, which, when bolted together, clamp a plastic tab on each thruster. These clamps provide a strong, secure mount that can be easily removed or adapted to other specifications.

The force that can be generated by the thruster is characterized, as shown in Fig. 3. The significant linearity between the thruster force and the applied duty cycle will significantly facilitate the design and implementation of any control scheme..

A fluid drag force model is established to evaluate the speed that the UUV can achieve. Fig. 4 shows the relationship between the drag force with respect to the relative velocity of the vehicle. Under the full load of the two thrusters, the vehicle is able to achieve a maximum forward or backward speed of $1.4 \mathrm{~m} / \mathrm{s}(\sim 5 \mathrm{~km} / \mathrm{hour})$. 


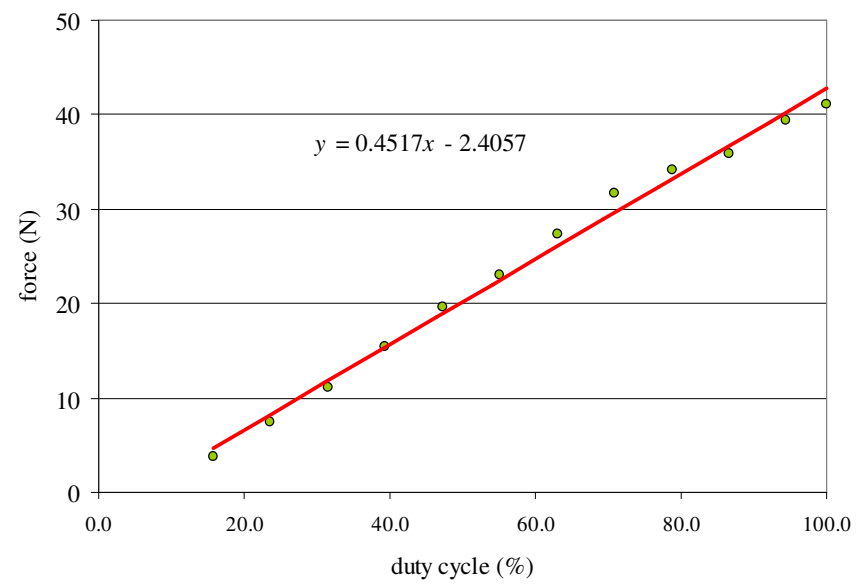

Fig. 3. Calibration of the motor: force with respect to duty cycle.



Fig. 4. Drag force of the UUV with different velocities.

\section{BAllast AND Depth CONTROL}

In the design, both static and dynamic diving principles are considered. Based on these criteria four initial concepts are developed [17]: i) a piston type ballast tank; ii) a hydraulic pump based ballast system; iii) an air compressor based system; and iv) direct thrust systems. The first three concepts come from static diving technology, while the last concept is a dynamic diving technology.

The piston ballast tank is one of the most common static diving methods applied in submarine modeling. A piston ballast tank consists of a cylinder and a movable piston, and it works as a large syringe pump. With one end of the cylinder connected to surrounding water, movement of the piston sucks water in or pushes it out. When water fills the tank, negative buoyancy is achieved, so the UUV starts to descend. Conversely, when the tank is emptied, the UUV is positively buoyant, so it ascends. This setup also allows control of pitch motions of the UUV. Moreover, the pistons can be moved by a linear actuator, which is electrically easy to control. Hence, accurate depth control can be achieved with proper, yet straightforward, programming.

A hydraulic pumping system is similar to the piston ballast tank, but uses an internal reservoir of hydraulic fluid 
and a pump to actuate the piston's linear motion. Control of the valves and the pump for the hydraulic fluid allows it to flow in and out of the cylinders, so the surrounding water can be pumped in and out. Consequently, buoyancy of the UUV is changed.

Air compressor systems are commonly used in some classes of submarine. The system is composed of a storage tank of compressed air, a water tank and two valves that are normally closed. To descend, the vent valve is opened, so the pressure difference results in water flowing in from the opening in the bottom of the water tank. When a desired amount of water is obtained for ballast, the vent valve is closed. In order to force the water out, the blow valve is opened to allow the compressed air into the tank so that water is pushed out via the bottom opening. Thus, by letting the water in and out of the water tank, the buoyancy of the UUV is changed.

Thrusters are a dynamic diving method. They require the UUV to be near neutrally buoyant. This approach uses the vertically mounted thrusters to force the UUV to dive. Turning off the thrusters or using them at a thrust less than the positive buoyancy allows controlled ascent. However, this method consumes a lot of power to keep the UUV under water, as the thrusters must remain powered at virtually all times. Being positively buoyant, however, this method is intrinsically failsafe, as the vehicle will come to the surface in the event of a power failure.

Selection of a suitable ballast system is dependent on various factors, such as design specifications, size and geometry of the UUV hull, depth required, and cost. In this design, the hull is made of a PVC pipe with an outer diameter of $400 \mathrm{~mm}$ and a length of $800 \mathrm{~mm}$. The required working depth is $20 \mathrm{~m}$. Hence, the ballast system selected not only has to meet the basic requirements enumerated above, but must also be able to fit in the hull. Preferably, all are at a relatively low cost.

First, installing two (2) $160 \mathrm{~mm}$ inner diameter ballast tanks of $250 \mathrm{~mm}$ length provides a net force of $\pm 5 \mathrm{~kg}$. Additionally, the force required to actuate the piston head at $20 \mathrm{~m}$ is calculated to be approximately $6000 \mathrm{~N}$. To generate such force on the piston head, a powerful linear actuator is needed. The specific linear actuator (LA36 24V DC input, 6800N max load, 250mm stoke length) can be sourced from Linak Ltd in New Zealand. However, the linear actuator has a duty cycle of $20 \%$ at max, which means that for every 20 s continuous work, it must remain off for 80 s before operating again, allowing the UUV to float uncontrolled. In addition, the cost of one linear actuator is US\$1036, which would imply that similar actuators with longer duty cycles would cost a larger amount at this time.

Taking the second option, a hydraulic pumping system can be customized from Scarlett Hydraulics Ltd, New Zealand. The overall system has dimensions of $500 \mathrm{~mm} \times 250 \mathrm{~mm} \times 250 \mathrm{~mm}$. It consists of a $1.2 \mathrm{KW}$ DC motor, a pump, a 4L hydraulic fluid tank, two dual solenoid valves and two cylindrical tanks. This system meets the required specifications, but has some drawbacks. In particular, it occupies too internal space of the hull, and weighs approximately $20 \mathrm{~kg}$ (a significant addition of weight). In this case, the overall hydraulic pumping system will cost up to approximately US $\$ 2264$.

The third option air compressor system is cost effective and is easy to operate by controlling the vent and blow valves. However, the lack of accuracy in controlling compressed gas is a major disadvantage. In addition, performance and operating time are limited by the amount of stored gas. In this design, a 10L tank would be 
TABLE I

BALLAST COMPARISON

\begin{tabular}{|c|c|c|c|c|c|c|}
\hline & Diving Tech & Installation & Buoyancy & Sealing & Reliability & $\begin{array}{l}\text { Overall Cost }{ }^{\dagger} \\
\text { (US\$) }\end{array}$ \\
\hline Piston ballast tanks & Static & Internal & $+\mathrm{ve}$, -ve, Neutral & Difficult & Used in most remote submarines & 2500 \\
\hline Hydraulic pumping system & Static & Internal & $+v e,-v e$, Neutral & Difficult & Not reliable & 2710 \\
\hline Air compressor & Static & Internal & $+v e$, -ve, Neutral & Difficult & $\begin{array}{l}\text { Air on board is limited, com- } \\
\text { pressed air hard to handle }\end{array}$ & 420 \\
\hline Thrusters & Dynamic & External & $+\mathrm{ve}$ & None & Used in most UUVs with big size & 500 \\
\hline
\end{tabular}

$\dagger$ The cost is estimated as an overall system.

needed to fulfil the changes in buoyancy. In other words, a gas cylinder containing $10 \mathrm{~L}$ of air compressed to at least 3 bar is required for a single diving and rising cycle. Hence, to refill the gas cylinder, the UUV must float to the waters surface before all the air runs out or risk being lost. Regarding the on-site requirement, that the UUV should operate for hours, the air tank must either be much bigger or far more highly pressurize, which leads to safety issues.

The fourth option thrusters are different from the previous three systems that all had to be installed inside the UUV. In contrast, thrusters can be attached externally. Hence, sealing is not as critical as it is for the other concepts. If the vehicle is trimmed positively buoyant, it is also reasonably fail-safe, unlike the other three methods. Additionally, the thrusters can be sourced from Pu Tuo Hai Qiang Ltd, Zhou Shan, China for US\$55/unit, a reduction of 12-20× in cost if two are used. Each thruster fits in a $215 \mathrm{~mm} \times 215 \mathrm{~mm} \times 80 \mathrm{~mm}$ box, and is driven by a $12 \mathrm{~V}$ DC motor with a max thrust force of $5 \mathrm{~kg}$ under water. By mounting the desired number of thrusters, a wide range of motions can be controlled, such as pitch and roll control. Finally, each concept has its own advantages and disadvantages. Comparisons are summarized in Table I. In this design, the major driving factors for the selection of ballast system are the cost and reliability. Piston ballast tank and thruster systems are reliable since these two depth control methods have been widely employed in most unmanned underwater vehicle development. Considering the cost, the thruster system is more effective. Hence, the thruster system is chosen as the final design.

There are four thrusters vertically mounted around the UUV with one at each corner (See Fig. 2). Mounting four thrusters produces a total of $20 \mathrm{~kg}$ thrust force at full load, and allows a wide range of motion control. They enable the control of not only the vertical up and down motion, but pitch and roll motions. To achieve this control, each thruster is connected to a speed control module that can be controlled via a central microprocessor. By inputting different digital signals, various forces thus speeds are generated. Therefore, desired motion control can be obtained by different combinations. 


\section{ELECTRONICS AND CONTROL}

\section{A. Power Supply}

For long term operation, this design must locate the power supply on-board, unlike many current models that receive power over an umbilical link [18]. Since all the systems onboard the UUV are electric, sealed lead acid batteries are chosen for the power supply. These batteries have high capacity and can deliver higher currents, than other types of rechargeable battery [19], [20]. They are stable, inexpensive, mechanically robust and can work in any orientation, all of which are important considerations in a vehicle of this type. To supply enough current for the entire machine several batteries have to be joined together. Instead of adding dead weight to achieve neutral buoyancy extra batteries can be added as needed so that the total operating time of the UUV is higher than that required for a given application.

It is also highly desirable to locate the battery compartments separate from the main hull so that they can be interchanged in the field without opening the sealed main hull. To accommodate this requirement two tubes are fitted below the hull to house batteries. Within these tubes the batteries are connected to two bus bars. Each battery is fused prior to connecting to the bus bar, and the bars are isolated to the greatest extent possible to increase safety. These bus bars are then wired into the main hull, where a waterproof socket enables the quick interchange of battery compartments. A similar bus system exists inside the hull with connections to motors and electronic power supplies. Each of these internal connections is similarly fused. Longer term, it would be desirable to intelligently monitor the bus to track the state of each battery and overall power consumption.

\section{B. Central Processing Unit}

The central processing unit is responsible for accessing sensors, processing data and setting control outputs such as motor speeds. Several systems are considered for this unit, an embedded system using microprocessors, FPGAs or a small desktop PC. A microprocessor system, most likely based on an ARM processor would have low cost, size and power requirements and is easy to interface to both analogue and digital sensors, motors and other actuators. The processing power and memory allocations of these microprocessors are all more than sufficient for the simple control tasks likely to be required, but would struggle with larger sensor or processing tasks, such as image processing. An FPGA system would also be small and have low power requirements, but would be more expensive. While FPGAs work very well for fast, complex processing tasks such as image processing, their complexity in design and programming necessitates their use in parallel with other more flexible CPU choices. The last system considered is a small desktop PC (Fig. 5). Although a desktop PC is bigger, more expensive and consumes more power than either of the prior two options, it provides immense processing power, memory and a diverse range of peripherals. It is therefore chosen in this initial design for the following primary reasons:

- Added power requirements were not an issue since we have a sizeable power supplies.

- Processing power is more than adequate for this initial design and future developments.

- Large volumes of memory are available, both volatile for program execution and solid state for storage of gathered data. 




Fig. 5. On board computer.

- Despite not having direct access to sensors and control units, a diverse range of peripherals available can be used, including USB, RS232 and Ethernet, enabling a potentially greater range of sensors and sensor platforms for developing broad ranges of specific applications.

- A USB module is already provided for a webcam for initial image sensing applications and an Ethernet module is provided for remote connection.

An AMD Sempron 3000+ processor and ASUS M2N-PV motherboard are used for this purpose. These models have lower power requirements and heat generation. Software interfaces this unit with sensors and motor controllers, as well as to a remote control PC. An automotive power supply (Exide, Auckland, NZ) is used to provide power for the computer. It takes a $12 \mathrm{~V}$ DC input and converts it to the ATX standard power supply required by the PC. This module is also designed to be used in an electrically noisy and hostile environment and is ideally suited the specific design situations considered.

\section{Sensors}

When the UUV is used autonomously, after development there will be a large and extensive sensor suite onboard. Currently, the sensors onboard measure

- water pressure, from which depth can be determined

- water temperature, inner hull temperature and humidity

- the UUV position in the three principal axes: yaw, pitch and roll

- visual or digital image feedback via a webcam.

Submersible pressure sensors that are salt water tolerant and can measure up to the pressures required are difficult to acquire at low cost. The sensor chosen was sourced from Mandeno Electronics for US\$121. This sensor measures up to twice the depth required, and outputs an analogue output between 0 and $100 \mathrm{mV}$. Thermocouples from Farnell Electronics (Christchurch, New Zealand) are used to measure the water temperature, and provide an analogue output relative to the temperature difference between the two ends of the thermocouple. TMP100 sensors (Texas Instruments) are used to measure the base temperature of the thermocouple, and the hulls interior temperature. These 


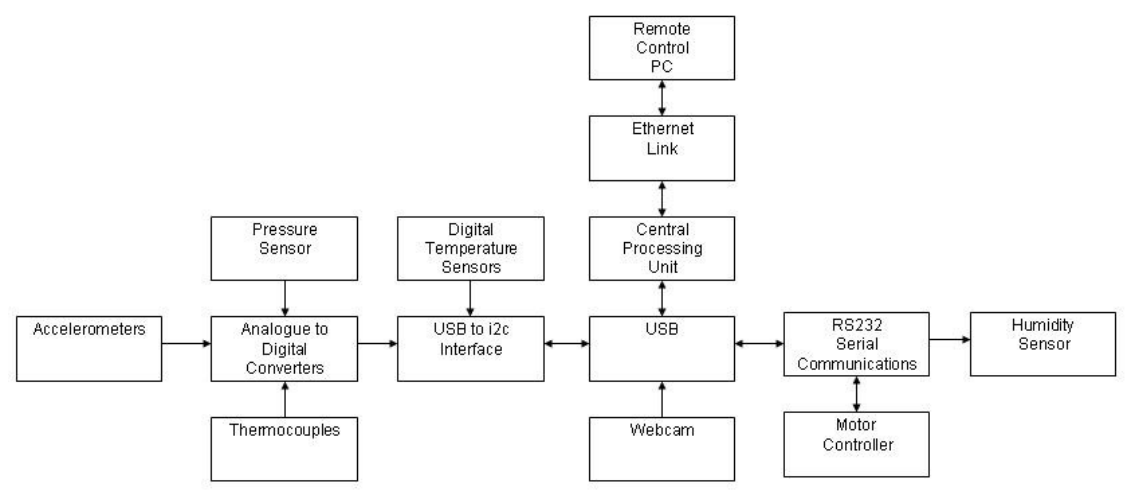

Fig. 6. The block diagram for electronic systems and control.

sensors give a digital output using the I2C protocol. A HF3223 humidity sensor (Digi-Key) is used to measure humidity inside the hull. A MMA7260QT accelerometer (Freescale Semiconductor) is used to calculate orientation. The accelerometer has a $0-2.5 \mathrm{~V}$ analogue output. The connection of the sensors is shown in Fig. 6 .

To eliminate signal noise, An Atmel AT90USB82 microprocessor is connected to the USB ports of the computer to move all noise sensitive data to the acquisition points. The analogue sensors are amplified using an INA2322 instrumentation amplifier, if necessary, and read by an ADS7828 analogue to digital converter. This converter is then connected to the Atmel microprocessor using a common I2C bus with the TMP100. The humidity sensor is attached to a clock input which converts the frequency based signal to a humidity based reading. The microprocessor performs some basic processing on this data, temperature compensating the pressure sensor and thermocouple, and calculating yaw, pitch and roll from the accelerometer readings.

Vidual or digital image sensing is included via a Logitech webcam connected directly to the on-board computers USB port. The video stream can be sent back over a wireless remote control network connection to the remote PC. At this stage, no image processing is done on this stream on-board, and it is included purely to assist in manual control of the UUV at this time, and for use in later application development.

\section{Propulsion Motor Driver}

For the six motors (two for horizontal propulsion, and four for vertical ballast control), three (3) RoboteQ AX2500 (Fig. 7) motor controllers are used for control. Each controller is able to control two motors up to 120 amps, much higher than the 25 amps needed by the motors selected. The controllers are controlled via RS232 (serial port) interfaces, which are already available on the computer motherboard. Computer control of the controllers is easily achieved through a LabView or MATLAB interface, either manually or automatically, where both interfaces have been implemented to allow greater user ease of use.

\section{E. Control System and Communications}

During testing and development, remote control is required for the UUV. Sensors readings need to be sent to a user, and control signals sent back to the UUV. Displaying the video feed from the webcam is also desired to 


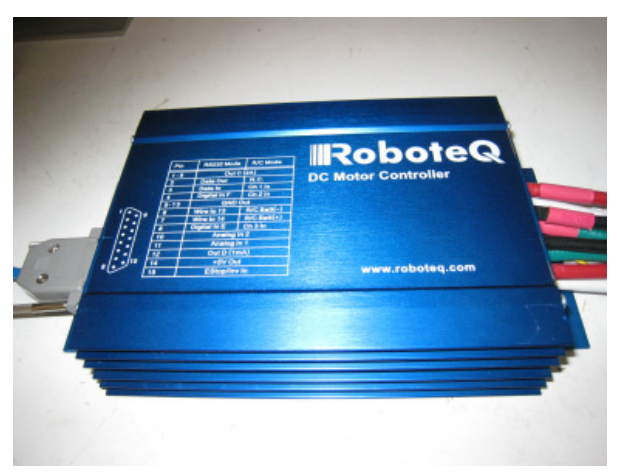

Fig. 7. The controller for both horizontal and vertical thrusters.

provide the operator with visual feedback. High frequency radio transmissions are impossible underwater due to the high losses encountered during the air/water boundary [21]. Lower frequency transmissions could have been used to communicate with the UUV, but they do not possess enough bandwidth to send the required data. An umbilical ethernet cable is being used for this remote link between the UUV and an external control computer for this development phase. Fig. 6 shows the electronics and control structure. Note that in an actual, developed application, or final development thereof, the robot will be acting autonomously and this umbilical will not be required.

\section{CONCLUSION}

A UUV has been successfully designed and prototyped for shallow water tasks, such as inspecting and cleaning sea chests of ships. It features low cost and wide potential use for normal shallow water tasks with a working depth up to $20 \mathrm{~m}$, and a forward/backward speed up to $1.4 \mathrm{~m} / \mathrm{s}$. Each part of the UUV is deliberately chosen based on a comparison of readily available low cost options when possible. The prototype has a complete set of components including vehicle hull, propulsion, depth control, sensors and electronics, batteries, and communications. The total cost for a one-off prototype is less than US\$10,000. With these elements, a full range of horizontal, vertical and rotational control of the UUV is possible including computer vision sensing. The overall underwater vehicle will be a good platform for research, as well as for its specific applications, many of which are growing in importance like the sea chest inspection case noted here.

\section{REFERENCES}

[1] D. Smallwood and L. Whitcomb, "Model-based dynamic positioning of underwater robotic vehicles: theory and experiment," IEEE Journal of Oceanic Engineering, vol. 29, no. 1, pp. 169-186, Jan. 2004.

[2] J. Horgan and D. Toal, "Review of machine vision applications in unmanned underwater vehicles," in 9th International Conference on Control, Automation, Robotics and Vision, Dec. 2006.

[3] M. Caccia, "Autonomous surface craft: prototypes and basic research issues," in 14th Mediterranean Conference on Control and Automation, June 2006.

[4] R. Wernli, "Low cost uuv's for military applications: Is the technology ready?" in Pacific Congress on Marine Science and Technology 2001, San Francisco, CA, July 2001. 
[5] C. Williams, "AUV systems research at the NRC-IOT: an update," in 2004 International Symposium on Underwater Technology, 2004, pp. 59-73.

[6] D. Smallwood, R. Bachmayer, and L. Whitecomd, "A new remotely operated underwater vehicle for dynamics and control research," in Proceedings UUST '99, 1999.

[7] G. Griffiths and I. Edwards, "AUVs: designing and operating next generation vehicles," Elsevier Oceanography Series, vol. 69, pp. 229-236, 2003.

[8] R. Ballard, The Discovery of the Titanic. New York, NY: Warner/Madison Press Books, 1987.

[9] M. Tivey, H. Johnson, A. Bradley, and D. Yoerger, "Thickness of a submarine lava flow determined from near-bottom magnetic field mapping by autonomous underwater vehicle," Geophysical Research Letters, vol. 25, pp. 805-808, 1998.

[10] H. Kondoa and T. Ura, "Navigation of an AUV for investigation of underwater structures," Control Engineering Practice, vol. 12, p. 1551C1559, 2004.

[11] S. Willcox, J. Vaganay, R. Grieve, and J. Rish, "The bluefin BPAUV: An organic wide-area bottom mapping and mine-hunting vehicle," in Proceedings UUST '01, 2001.

[12] T. Curtin and J. Bellingham, "Autonomous ocean-sampling networks," IEEE Journal of Oceanic Engineering, vol. 26, pp. 421-423, 2001.

[13] J. Rife and S. M. Rock, "Field experiments in the control of a jellyfish tracking ROV," in MTS/IEEE Oceans '02, vol. 4, 2002, pp. 2031-2038.

[14] J. Lygouras, K. Lalakos, and P. Tsalides, "THETIS: an underwater remotely operated vehicle for water pollution measurements," Microprocessors and Microsystems, vol. 22, no. 5, pp. 227-237, Sept. 1998.

[15] R. Uhrich and S. Watson, "Deep-ocean search and inspection: Advanced unmanned search system (AUSS) concept of operation," Naval Command, Control and Ocean Surveillance Center, RDT\&E Division, San Diego, CA, Tech. Rep. NRaD TR 1530, Nov. 1992.

[16] E. Allmendinger, Submersible Vehicle Systems Design. Jersey City, NJ: Society of Naval Architects and Maringe Engineers, 1990.

[17] M. Wolf, "The design of a pneumatic system for a small scale remotely operated vehicle," Bachalor's Thesis, MIT, May 2003.

[18] Y. Chardard and T. Copros, "Swimmer: final sea demonstration of this innovative hybrid AUV/ROV system," in Proceedings 2002 International Symposium on Underwater Technology, Tokyo, Japan, Apr. 2002, pp. 17-23.

[19] G. Schubak and D. Scott, "A techno-economic comparison of power systems for autonomous underwater vehicles," IEEE Journal of Oceanic Engineering, vol. 20, no. 1, pp. 94-100, 1995.

[20] A. Bradley, M. Feezor, H. Singh, and F. Sorrell, "Power systems for autonomous underwater vehicles," IEEE Journal of Oceanic Engineering, vol. 26, no. 4, pp. 526-538, 2001.

[21] A. Leonessa, J. Mandello, Y. Morel, and M. Vidal, "Design of a small, multi-purpose, autonomous surface vessel," in Proceedings OCEANS 2003, vol. 1, San Diego, CA, USA, 2003, pp. 544-550. 